\title{
Receiver Design for Molecular Communication
}

\author{
Deniz Kilinc, Student Member, IEEE, Ozgur B. Akan, Senior Member, IEEE
}

\begin{abstract}
In the Molecular Communicaiton (MC), molecules are utilized to encode, transmit, and receive information. Transmission of the information is achieved by means of diffusion of molecules and the information is recovered based on the molecule concentration variations at the receiver location. The MC is very prone to intersymbol interference (ISI) due to residual molecules emitted previously. Furthermore, the stochastic nature of the molecule movements adds noise to the MC. For the first time, we propose four methods for a receiver in the MC to recover the transmitted information distorted by both ISI and noise. We introduce sequence detection methods based on maximum a posteriori (MAP) and maximum likelihood (ML) criterions, a linear equalizer based on minimum mean-square error (MMSE) criterion, and a decision-feedback equalizer (DFE) which is a nonlinear equalizer. We present a channel estimator to estimate time varying MC channel at the receiver. The performances of the proposed methods based on bit error rates are evaluated. The sequence detection methods reveal the best performance at the expense of computational complexity. However, the MMSE equalizer has the lowest performance with the lowest computational complexity. The results show that using these methods significantly increases the information transmission rate in the MC.
\end{abstract}

Index Terms-Molecular communication, sequence detection, channel equalization, signal-dependent noise, intersymbol interference.

\section{INTRODUCTION}

Molecular communication (MC) is a promising paradigm for communication at nanoscale [1], [2] and it is inspired by the communication of the biological systems at cellular level [3]. The construction of nanonetworks by interconnecting nanodevices via MC channels expands the capabilities of single nanodevices by means of cooperation between them [4], [5]. In the MC, molecules are utilized to encode, transmit, and receive information. It is believed that the integration process of the molecular transceivers in nanodevices is more feasible due to their size and natural domain unlike the classical communication techniques [6]. In the MC, the information transmission relies on the diffusion process, i.e., the propagation of molecules by means of the laws of free diffusion in an aqueous medium, enabling the transmission of the information. Several nature-inspired and theoretically modeled MC techniques exist in the literature. For example, in [7], based on the diffusion of pheromones, spores, and pollen, a long range interconnection of nanodevices deployed over

Manuscript received August 1, 2012; revised November 4, 2012; accepted March 1, 2013.

The authors are with the Next-generation and Wireless Communications Laboratory (NWCL), Department of Electrical and Electronics Engineering, Koc University, Istanbul, 34450, Turkey (e-mail: \{dkilinc, akan\}@ku.edu.tr)

This work was supported in part by the Turkish Scientific and Technical Research Council under grant \#109E257, by the Turkish National Academy of Sciences Distinguished Young Scientist Award Program (TUBA-GEBIP), and by IBM through IBM Faculty Award. distances from a few centimeters to several meters is discussed. On the other hand, short range MC channels based on gap junction channels between cardiomyocytes can be constructed [8]. In [4], flagellated bacteria and catalytic nanomotors are two proposed communication techniques for the transport of DNA encoded information between emitters and receivers by means of a physical carrier. Morphogenesis is also proposed as a method for communication of nanodevices [9].

The research on the information theoretical investigation of the MC has been heavily conducted in the literature [10], [11], [12], [13]. However, to the date, there is little effort addressing the design of a receiver nanodevice which detects the transmitted information from the received concentration signal. Although in [14], [15], the diffusion-based MC channel is analyzed from an information theoretical perspective, noise sources are not incorporated to the analysis. For the first time, the noise sources in the MC are analyzed in [6].

For the MC, the information is encoded in the concentration of the emitted molecules with on-off keying (OOK) modulation in [14], [15], [16]. As described in [10], an emitted concentration pulse changes the molecule concentration at the receiver location. Because of the diffusion of the emitted molecules from regions of high concentration to those of low concentration, the molecule concentration at the receiver location decreases if the molecule emission ceases. However, the emitted molecules do not vanish completely in the reception space. That is, if a new concentration pulse is emitted before the molecule concentration at the receiver location becomes negligibly low, the receiver senses the effects of both the previously emitted concentration pulses and the currently transmitted pulse. Therefore, a low signaling interval, i.e., a high transmission rate, causes intersymbol interference (ISI) in the MC. If the transmitter waits long enough to send a new concentration pulse, the effect of the ISI can be eliminated; then, the information transmission rate becomes very low. Nevertheless, having high transmission rates is desirable to improve communication capabilities of nanodevices.

For the first time in the literature, in order to recover the transmitted information distorted by both the ISI and the noise at high transmission rates in the MC, we propose the following solutions. Firstly, the sequence detection methods based on maximum a posteriori (MAP) and maximum likelihood (ML) criterions are presented. Secondly, we present a linear equalizer based on minimum mean-square error (MMSE). Finally, a decision-feedback equalizer (DFE) is proposed. These methods are well covered for the classical communication systems [17], [18]. However, most of these techniques are developed for the communication channels having additive white Gaussian noise (AWGN) at the output of the channel. Since in the MC, the noise that is added to the received signal is signal-dependent which makes the noise nonstationary [6], 
the existing techniques in the classical communication cannot be applied directly to the MC. In this paper, we modify the detection methods stated above to properly implement for a receiver in the MC. The proposed methods significantly decrease the bit error probabilities and hence increase the communication capabilities of nanodevices communicating via MC. Since the magnetic recording channels have also signaldependent noise at the output of the channel [19], [20], the signal detection methods used for magnetic recording channels are useful in the design of a receiver for the MC.

The rest of the paper is organized as follows. Section II describes the model of the MC. In Section III, the sequence detection methods based on MAP and ML criterions are presented. A linear equalizer which satisfies the MMSE criterion is described in Section IV. A nonlinear type of equalizer which is a decision-feedback equalizer is presented in Section V. In Section VI, to estimate the time varying channel, a channel estimator is presented. The performance of these methods based on bit error rates are evaluated in Section VII. Finally, Section VIII concludes the paper.

\section{Molecular Communication Model}

The MC model that is considered in this paper consists of a Transmitter Nanodevice (TN), molecular diffusion channel, signal-dependent additive noise at the output of the channel, and a Receiver Nanodevice (RN) as illustrated in Fig. 1. TN encodes the binary information bits, $\left\{a_{j}\right\}$, into the molecule concentration pulses. The concentration pulse, denoted by $s(t)$, is the time variation of the molecule concentration emitted by $\mathrm{TN}$ for the transmission of one information bit. The transmitted concentration signal propagates until it reaches the receiver location. The propagation relies on the particle diffusion process in the aqueous medium. At the reception space, the molecule concentration, denoted by $y(t)$, changes according to both the time and the distance between TN and RN. Furthermore, a signal-dependent concentration noise, $n(t)$, is added to the molecule concentration signal in the reception space. RN samples the noisy molecule concentration, denoted by $z(t)$, in the reception space, and decides the transmitted information sequence based on these samples. In the model, we consider following assumptions:

- The communication takes place in a 3D aqueous environment having infinite extent which is indexed through the Cartesian axes $x, y$, and $z$.

- The size of the TN is considered negligible with respect to the distance between the TN and the RN. Therefore, the $\mathrm{TN}$ is approximated as a point source emitting desired molecule concentration signals at the location $(x=0, y=0, z=0)$.

- The emitted molecules by TN propagates from the TN location $(x=0, y=0, z=0)$ to the $\mathrm{RN}$ location $\left(x=x_{R}, y=y_{R}, z=z_{R}\right)$ only by means of the laws of free diffusion in an aqueous medium. That is, the emitted molecules are subject to the Brownian motion.

- The particle concentration measurement takes place inside the reception space having a spherical shape with radius $\rho$. The received molecule concentration, $z(t)$, is assumed homogeneous inside the reception space.

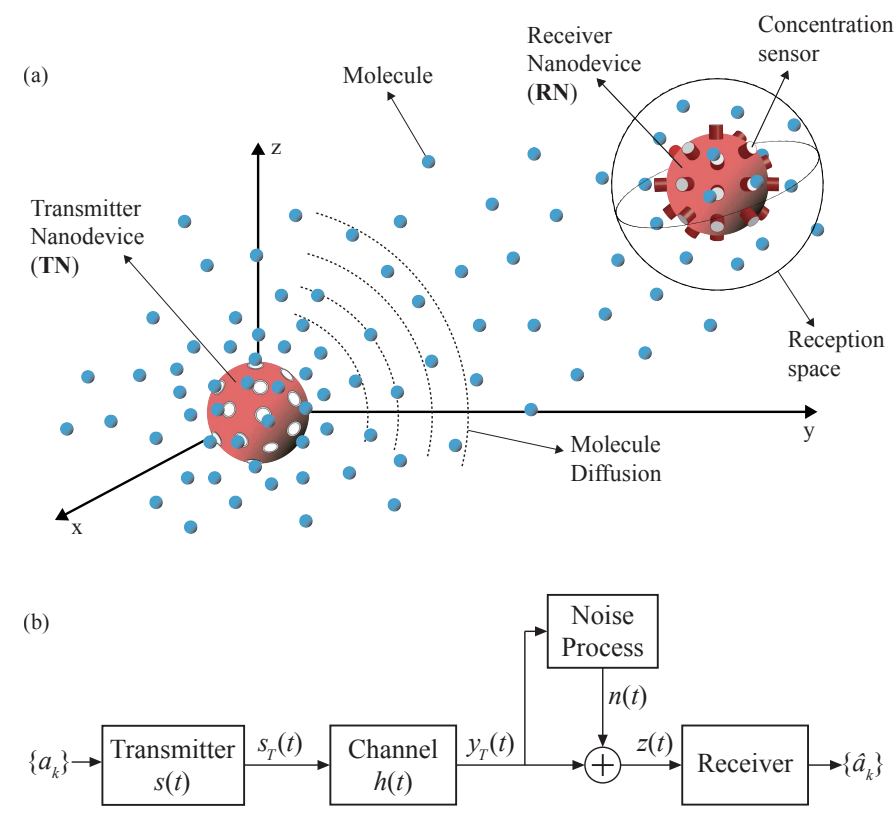

Fig. 1. The diffusion-based MC model between TN and RN. (a) Graphical representation. (b) Block diagram representation.

- The communication between TN and RN is considered as the binary digital communication with OOK modulation with equally likely transmitted binary information bits.

Firstly, we derive the impulse response of the molecular diffusion channel, i.e., $h(t)$. According to Fick's second law of diffusion [14], the concentration of the molecules, denoted by $c(x, y, z, t)$, at location $(x, y, z)$ and at time $t$ is described by the following equation

$$
\frac{1}{D} \frac{\partial c(x, y, z, t)}{\partial t}=\nabla^{2} c(x, y, z, t)
$$

where $D$ is the diffusion coefficient of the medium and $\nabla^{2} c(x, y, z, t)$ is the sum of the 3D spatial second derivatives of $c(x, y, z, t)$. The impulse response of the molecular diffusion channel is found by solving (1) with initial condition

$$
c(x, y, z, t=0)=\delta(x, y, z)
$$

where the molecules are emitted by $\mathrm{TN}$ which is a point source located at $(x=0, y=0, z=0)$. The response of (1) to the impulse given in (2) is

$$
c(x, y, z, t)=\frac{m}{(4 \pi t D)^{3 / 2}} \exp \left[-\frac{(x)^{2}+(y)^{2}+(z)^{2}}{4 t D}\right] .
$$

where $m$ is the total number of the emitted molecules. Therefore, in a $3 \mathrm{D}$ medium, if $\mathrm{RN}$ is located at $\left(x_{R}, y_{R}, z_{R}\right)$, the impulse response of the diffusion channel $h(t)$ is given by

$$
h(t)=\frac{m}{(4 \pi t D)^{3 / 2}} \exp \left[-\frac{|r|^{2}}{4 t D}\right]
$$

where $|r|$ is the Euclidean distance between RN and TN, i.e., $|r|=\sqrt{\left(x_{R}\right)^{2}+\left(y_{R}\right)^{2}+\left(z_{R}\right)^{2}}$. To have a compact notation, we drop $(x, y, z)$ term in $h(x, y, z, t)$ because $\mathrm{TN}$ and $\mathrm{RN}$ are located at fixed positions. Since the diffusion coefficient $D$ depends on several factors such as the temperature and 
viscosity of the fluid which are time varying properties, the diffusion-based molecular channel is also time varying.

We consider the transmitter concentration pulse as a rectangular pulse given as

$$
s(t)=\operatorname{Arect}\left(\frac{t-T_{e} / 2}{T_{e}}\right)
$$

where $A$ is the amplitude of the molecule concentration emitted by $\mathrm{TN}$ and $T_{e}$ is the duration of the molecule emission. Therefore, the response of the channel to the input signal $s(t)$ is denoted by $y(t)$ and can be obtained using the convolution operation as $y(t)=s(t) * h(t)$. The physical meaning of $y(t)$ is the noiseless molecule concentration variation in the reception space caused by one emitted pulse. The variation of $y(t)$ with respect to time is shown in Fig. 2. Assuming the communication starts at time $t=0$, the total emitted molecule concentration into the aqueous environment, denoted by $s_{T}(t)$, for the transmitted information sequence $\left\{a_{j}\right\}$ is given as

$$
s_{T}(t)=\sum_{j=0}^{\infty} a_{j} s(t-j T)
$$

where $T$ is the duration of the signaling interval. Since the response of the channel to the pulse $s(t)$ is given by $y(t)$, the total noiseless received concentration by $\mathrm{RN}$ is

$$
y_{T}(t)=\sum_{j=0}^{\infty} a_{j} y(t-j T)
$$

The emission of a new pulse before the previously emitted molecules vanish in the reception space causes ISI because $y(t)$ is a infinite duration signal and do not disappear completely in the reception space in a short time as illustrated in Fig 2. That is, the currently emitted pulse is distorted due to the previous transmissions which can be seen in (7). Since $y(t)$ decreases after reaching its maximum value at $t=t_{\max }$, the signaling interval $T$ should satisfy the condition $T \gg t_{\max }$ in order to have negligible ISI. However, an increase in the signaling interval decreases the transmission rate given as $R=1 / T$ and a low information transmission rate is undesirable. It is aimed to have high transmission rates to improve the communication capabilities of nanodevices using the MC. As a result, to deal with the distortion caused by the ISI at higher transmission rates, we introduce several methods for RN which are discussed in the subsequent sections.

At the output of the channel, an additive signal-dependent noise is added to the ISI distorted signal $y_{T}(t)$. Then, the total molecule concentration variation in the reception space caused by the transmitted information sequence $\left\{a_{j}\right\}$ is given by

$$
z(t)=\sum_{j=0}^{\infty} a_{j} y(t-j T)+n(t)
$$

where $n(t)$ is the particle counting noise generated by a random process [6]. To describe the noise introduced in [6], firstly, we need to define the noiseless and noisy number of molecules inside the reception space, denoted by $N_{p}(t)$ and $\hat{N}_{p}(t)$, respectively, given as

$$
N_{p}(t)=V_{R} y_{T}(t) ; \quad \hat{N}_{p}(t)=V_{R} z(t)
$$

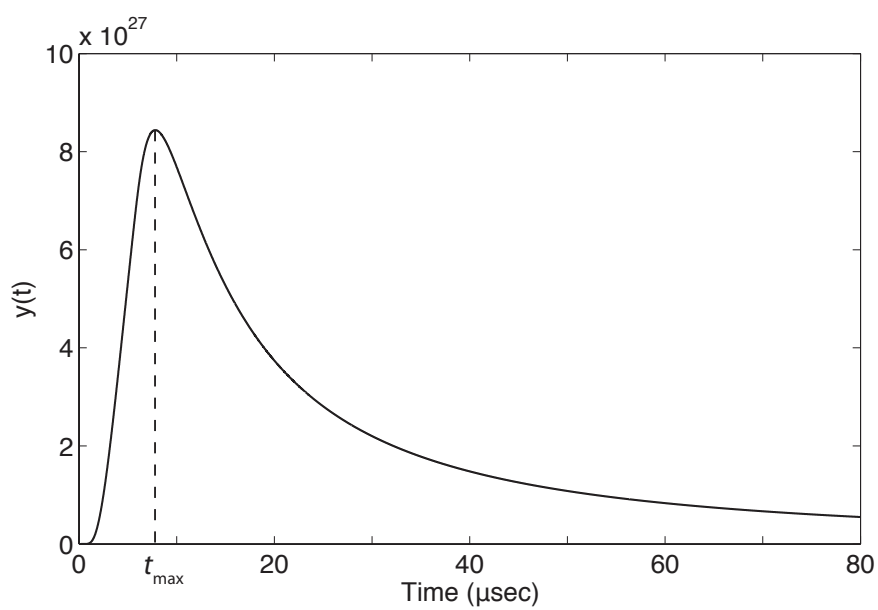

Fig. 2. The noiseless molecule concentration variation at $\mathrm{RN}, \mathrm{y}(\mathrm{t})$, for $D=$ $2.2 \times 10^{-9} m^{2} / s, r=250 \mathrm{~nm}, m=10^{5}, A=4 \times 10^{9}, T_{e}=4.7 \mu s$.

where $V_{R}=(4 / 3) \pi \rho^{3}$ is the volume of the spherical reception space. As given in [6], $\hat{N}_{p}(t)$ is distributed with Poisson probability distribution function (pdf) whose parameter is $N_{p}(t)$ at observation time $t$. Therefore, both the expectation and variance of the number of molecules in the reception space is $N_{p}(t)$. Furthermore, in a reliable MC between TN and $\mathrm{RN}$, we can assume that $N_{p}(t) \gg 100$ condition is satisfied at any instant. Thus, the Gaussian approximation of the Poisson distribution for $\hat{N}_{p}(t)$ can be used, i.e., $\hat{N}_{p}(t) \sim \mathcal{N}\left(N_{p}(t), N_{p}(t)\right)$. By using (9) and the pdf of $\hat{N}_{p}(t)$, the received noisy molecule concentration in the reception space, $z(t)$, is distributed as $z(t) \sim \mathcal{N}\left(y_{T}(t), y_{T}(t) / V_{R}\right)$. Finally, the probability distribution of the noise, $n(t)$, is given as $n(t) \sim \mathcal{N}\left(0, y_{T}(t) / V_{R}\right)$. Thus, the variance of the additive Gaussian noise $n(t)$ depends on both $y(t)$ and the transmitted information sequence $\left\{a_{j}\right\}$ which makes the noise nonstationary. Furthermore, based on the independency of the Brownian movement of different molecules in the aqueous medium, the noise samples are assumed independent of each other at different observation times [6].

We assume that $\mathrm{RN}$ is able to sample the molecule concentration inside the reception space at a rate $R$, and the TN and $\mathrm{RN}$ are synchronized, i.e., both transmission and reception start at the same time $t=0$. Therefore, the received signal samples by the $\mathrm{RN}$ is given as

$$
z_{k}=\sum_{j=0}^{\infty} a_{j} y_{k-j}+n_{k}, \quad k=0,1, \ldots
$$

where $z_{k}=z(k T), y_{k-j}=y((k-j) T)$, and $n_{k}=n(k T)$. However, the channel response is causal, i.e., $y_{k}=0$ if $k<0$. Furthermore, in a practical system, it is acceptable to assume that the length of the ISI is finite and given by $I+1$; that is, $y_{k}=0$ for $k>I$. Thus, $z_{k}$ depends on the currently transmitted bit $a_{k}$ and $I$ previously transmitted bits $a_{k-1}, \ldots, a_{k-I}$. Then,

$z_{k}=\sum_{j=k-I}^{k} a_{j} y_{k-j}+n_{k}=\sum_{j=0}^{I} y_{j} a_{k-j}+n_{k}, \quad k=0,1, \ldots$ 


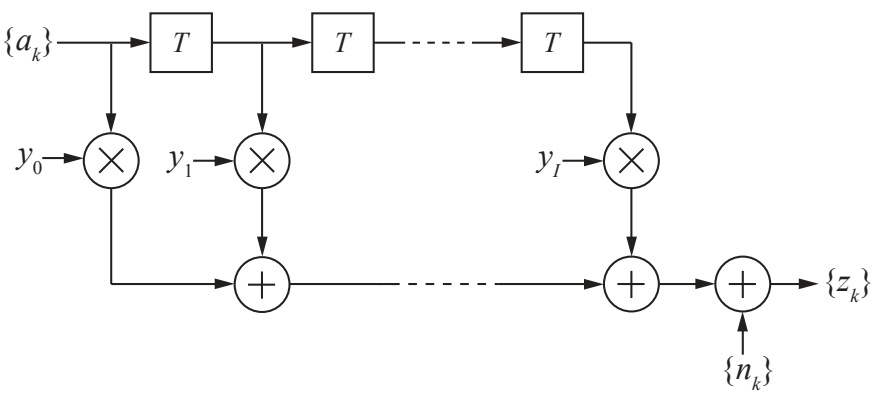

Fig. 3. Discrete-time equivalent model of the molecular diffusion channel with intersymbol interference.

Using (11), the discrete-time equivalent model of the diffusionbased MC channel is shown in Fig. 3. The pdf of $z_{k}$ is

$$
z_{k} \sim \mathcal{N}\left(\mu_{k}, \sigma_{k}^{2}\right)
$$

where the mean and variance, respectively, are

$$
\mu_{k}=\sum_{j=0}^{I} y_{j} a_{k-j} ; \quad \sigma_{k}^{2}=\frac{1}{V_{R}} \sum_{j=0}^{I} y_{j} a_{k-j}
$$

and the pdf of the noise samples is given as

$$
n_{k} \sim \mathcal{N}\left(0, \sigma_{k}^{2}\right)
$$

After describing the propagation and noise models of the diffusion-based $\mathrm{MC}$, we present several methods for $\mathrm{RN}$ to recover the transmitted information bits that are distorted by both the ISI and noise.

\section{Sequence Detection}

In the presence of the ISI, the sequence detection is equivalent to the problem of estimating the state of a finite-state machine (FSM) [17]. For ISI with length $I+1$, the state of the FSM at any instant depends on the $I$ most recent inputs. Since we consider binary communication, the channel has $2^{I}$ states. Therefore, the channel can be described by a $2^{I}$-state trellis and the transmitted information sequence corresponds to a path through the trellis. In Fig. 4, a trellis is illustrated for $I=2$. Each branch represents a state transition having a weight called the branch metric. The sum of the weights of each branch in a path is called the path metric. A sequence detector selects a path (sequence) corresponding to the received signal sequence $\left\{z_{k}\right\}$ based on the path metric of the each possible path, which is discussed later in this section. We consider two criterions to obtain the branch metrics: the maximum a posteriori (MAP) and maximum likelihood (ML) where the former selects the sequence maximizing the a posteriori probability density function and the latter selects the sequence maximizing the likelihood function in the trellis search. Furthermore, RN can reduce the complexity of the sequence detector and the number of sequences in the trellis search by using the Viterbi algorithm to discard the unlikely sequences when the new samples are received by the RN [21].

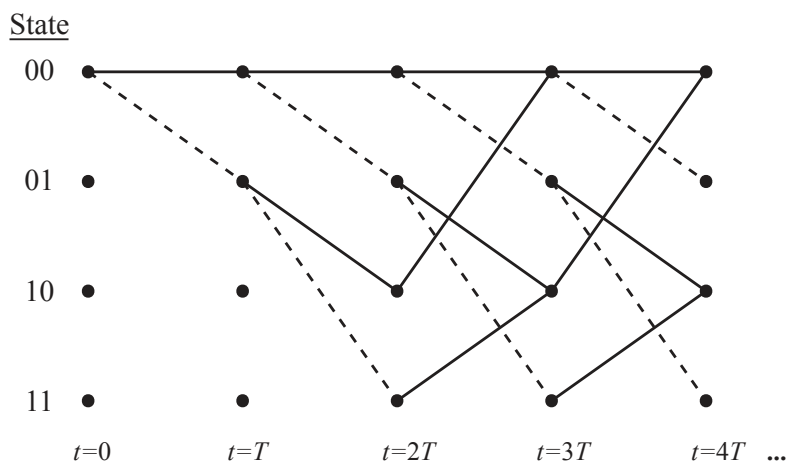

Fig. 4. A trellis diagram for $I=2$ with $2^{I}$ states.

\section{A. MAP Sequence Detection}

The MAP sequence detector determines the transmitted sequence of information bits $\left\{a_{j}\right\}$ by maximizing the joint pdf of the transmitted bits and the received samples [19]. That is,

$$
\underline{\hat{\boldsymbol{a}}}_{0}^{N}=\arg \max _{\underline{\boldsymbol{a}}_{0}^{N}} f\left(\underline{\boldsymbol{z}}_{0}^{N}, \underline{\boldsymbol{a}}_{0}^{N}\right)
$$

where $N+1$ is the length of the transmitted sequence of bits, $\underline{\hat{\boldsymbol{a}}}_{0}^{N}$ denotes the detected sequence for the transmitted information sequence $\underline{\boldsymbol{a}}_{0}^{N}$, the pdf $f\left(\underline{\boldsymbol{z}}_{0}^{N}, \underline{\boldsymbol{a}}_{0}^{N}\right)$ is the joint pdf of the received concentration signal samples $\underline{z}_{0}^{N}$ and the transmitted bits $\underline{\boldsymbol{a}}_{0}^{N}$. The bold and underlined symbols indicate the sequence of those symbols whose starting and ending elements are indicated in the subscript and superscript indexes, respectively. By Bayes rule, the joint pdf can be factored as follows

$$
\begin{aligned}
f\left(\underline{\boldsymbol{z}}_{0}^{N}, \underline{\boldsymbol{a}}_{0}^{N}\right) & =\mathrm{P}\left(\underline{\boldsymbol{a}}_{0}^{N}\right) f\left(\underline{\boldsymbol{z}}_{0}^{N} \mid \underline{\boldsymbol{a}}_{0}^{N}\right) \\
& =\prod_{k=0}^{N} \mathrm{P}\left(a_{k} \mid \underline{\boldsymbol{a}}_{k+1}^{N}\right) \prod_{k=0}^{N} f\left(z_{k} \mid \underline{\boldsymbol{z}}_{k+1}^{N}, \underline{\boldsymbol{a}}_{0}^{N}\right)
\end{aligned}
$$

where $\mathrm{P}\left(\underline{\boldsymbol{a}}_{0}^{N}\right)$ and $\mathrm{P}\left(a_{k} \mid \underline{\boldsymbol{a}}_{k+1}^{N}\right)$ are the joint and conditional probability mass functions of the transmitted binary information bits, respectively, $f\left(\underline{\boldsymbol{z}}_{0}^{N} \mid \underline{\boldsymbol{a}}_{0}^{N}\right)$ and $f\left(z_{k} \mid \underline{\boldsymbol{z}}_{k+1}^{N}, \underline{\boldsymbol{a}}_{0}^{N}\right)$ are the conditional pdf's. Since $a_{k}$ 's are assumed as independent,

$$
\mathrm{P}\left(a_{k} \mid \underline{\boldsymbol{a}}_{k+1}^{N}\right)=\mathrm{P}\left(a_{k}\right)
$$

The noise samples are also independent and the channel has a finite ISI length; thus, we can exploit these properties of the MC to simplify (16). The conditional pdf of each observation can be further simplified as follows

$$
f\left(z_{k} \mid z_{k+1}^{N}, \underline{\boldsymbol{a}}_{0}^{N}\right)=f\left(z_{k} \mid \underline{\boldsymbol{a}}_{k-I}^{k}\right)
$$

The conditional pdf of $z_{k}$ depends only on $\underline{\boldsymbol{a}}_{k-I}^{k}$ which also can be seen in (11).

Maximizing the joint pdf given in (16) is equivalent to minimizing its negative logarithm. Therefore, the MAP detector 
is given as

$$
\begin{aligned}
\underline{\hat{\boldsymbol{a}}}_{0}^{N} & =-\arg \min _{\underline{\boldsymbol{a}}_{0}^{N}} \ln \left[\prod_{k=0}^{N} \mathrm{P}\left(a_{k}\right) \prod_{k=0}^{N} f\left(z_{k} \mid \underline{\boldsymbol{a}}_{k-I}^{k}\right)\right] \\
& =-\arg \min _{\underline{\boldsymbol{a}}_{0}^{N}}\left[\sum_{k=0}^{N} \ln \mathrm{P}\left(a_{k}\right)+\sum_{k=0}^{N} \ln f\left(z_{k} \mid \underline{\boldsymbol{a}}_{k-I}^{k}\right)\right] \\
& =-\arg \min _{\underline{\boldsymbol{a}}_{0}^{N}} \sum_{k=0}^{N}\left[\ln \mathrm{P}\left(a_{k}\right)+\ln f\left(z_{k} \mid \underline{\boldsymbol{a}}_{k-I}^{k}\right)\right] \\
& =\arg \min _{\underline{\boldsymbol{a}}_{0}^{N}} \sum_{k=0}^{N} \mathcal{M}^{\mathrm{MAP}}\left(z_{k}, \underline{\boldsymbol{a}}_{k-I}^{k}\right)
\end{aligned}
$$

where $\mathcal{M}^{\mathrm{MAP}}\left(z_{k}, \underline{\boldsymbol{a}}_{k-I}^{k}\right)$ is the branch metric of trellis search in the Viterbi algorithm for the MAP criterion and given by

$$
\mathcal{M}^{\mathrm{MAP}}\left(z_{k}, \underline{\boldsymbol{a}}_{k-I}^{k}\right)=-\ln \mathrm{P}\left(a_{k}\right)-\ln f\left(z_{k} \mid \underline{\boldsymbol{a}}_{k-I}^{k}\right)
$$

By using the pdf of the noise samples, the conditional pdf of the concentration samples is given as

$$
f\left(z_{k} \mid \underline{\boldsymbol{a}}_{k-I}^{k}\right)=\frac{1}{\sqrt{2 \pi \sigma_{k}^{2}}} \exp \left[-\frac{\left(z_{k}-\mu_{k}\right)^{2}}{2 \sigma_{k}^{2}}\right]
$$

where $\mu_{k}$ and $\sigma_{k}^{2}$ are given in (13). After substituting the pdf given in (21) into the expression for the branch metric (20), and eliminating constant terms that are common, the MAP branch metric becomes

$$
\mathcal{M}^{\mathrm{MAP}}\left(z_{k}, \underline{\boldsymbol{a}}_{k-I}^{k}\right)=\ln \sigma_{k}^{2}+\frac{\left(z_{k}-\mu_{k}\right)^{2}}{\sigma_{k}^{2}}-2 \ln \mathrm{P}\left(a_{k}\right)
$$

\section{B. ML Sequence Detection}

The ML sequence detection method determines the transmitted sequence of information bits $\left\{a_{j}\right\}$ by maximizing the likelihood function [17], i.e.,

$$
\underline{\hat{\boldsymbol{a}}}_{0}^{N}=\arg \max _{\underline{\boldsymbol{a}}_{0}^{N}} f\left(\underline{\boldsymbol{z}}_{0}^{N} \mid \underline{\boldsymbol{a}}_{0}^{N}\right)
$$

where the likelihood function $f\left(\underline{\boldsymbol{z}}_{0}^{N} \mid \underline{\boldsymbol{a}}_{0}^{N}\right)$ is the joint pdf of the received concentration signal samples $\underline{\boldsymbol{z}}_{0}^{N}$ conditioned on the transmitted bits $\underline{\boldsymbol{a}}_{0}^{N}$. Furthermore, if the transmitted binary information bits are equally probable, i.e., $\mathrm{P}\left(a_{k}\right)=\frac{1}{2}$, the MAP sequence detection is identical to the ML sequence detection. The ML sequence detector is the optimum detector minimizing the error probability of a sequence [17]. With arguments similar to those for MAP sequence detection, the ML sequence detection can be simplified as follows

$$
\underline{\hat{\boldsymbol{a}}}_{0}^{N}=\arg \min _{\underline{\boldsymbol{a}}_{0}^{N}} \sum_{k=0}^{N} \mathcal{M}^{\mathrm{ML}}\left(z_{k}, \underline{\boldsymbol{a}}_{k-I}^{k}\right)
$$

where $\mathcal{M}^{\mathrm{ML}}\left(z_{k}, \underline{\boldsymbol{a}}_{k-I}^{k}\right)$ is the branch metric of the trellis search for the ML criterion. By using (22) and eliminating the constant terms, the ML branch metric is

$$
\mathcal{M}^{\mathrm{ML}}\left(z_{k}, \underline{\boldsymbol{a}}_{k-I}^{k}\right)=\ln \sigma_{k}^{2}+\frac{\left(z_{k}-\mu_{k}\right)^{2}}{\sigma_{k}^{2}}
$$

A similar result is found in [20] for the magnetic recording channels which have also signal dependent noise. After deriving the branch metrics, the Viterbi algorithm can be easily implemented to reduce the computational complexity of the MAP and ML sequence detection methods.

\section{Minimum Mean-Square Error Equalizer}

The MAP sequence detection in the presence of a priori symbol probabilities and the ML detection in the presence of equally probable symbols minimize the probability of a sequence error [17]. However, the complexities of the MAP and ML detectors grow exponentially with an increase in the length of the ISI even if the Viterbi algorithm is implemented. At nanoscale, the implementation of a complex circuitry is not practical. In this section, to mitigate the ISI, we present a linear equalizer based on the minimum mean-square error (MMSE) criterion called MMSE equalizer. The MMSE equalizer is a suboptimum linear equalizer with significantly less complexity compared to the MAP and ML sequence detectors [17].

In the MMSE equalization, the equalizer is designed to minimize the mean-square error (MSE), i.e., $\varepsilon_{k}=a_{k-d}-\tilde{a}_{k}$ where $a_{k-d}$ is the transmitted bit in the $(k-d)$ th signaling interval, $\tilde{a}_{k}$ is the estimate of that bit at the output of the equalizer and $d$ is the equalizer delay. The equalizer delay is found as follows. Let $y_{d_{1}}$ be the greatest magnitude component of $\left\{y_{k}\right\}$. Also, let the number of MMSE equalizer taps be equal to $S=2 d_{2}+1$ where $d_{2}$ is an integer. Then, we choose the equalizer delay as $d=d_{1}+d_{2}$ [22]. Since the MMSE equalizer is linear, its output as a linear combination of input samples is given by

$$
\tilde{a}_{k}=\sum_{q=0}^{S-1} r_{q} z_{k-q}
$$

where $r_{0}$ through $r_{S-1}$ are tap weight coefficients of the MMSE equalizer as illustrated in Fig. 5. The output $\tilde{a}_{k}$ is quantized to the nearest information bit in Euclidian distance to decide $\hat{a}_{k}$ by using a simple quantizer. We assume that the number of taps of the MMSE equalizer is the same as the ISI length, i.e., $S=I+1$.

To minimize the MSE which is defined as $J=\mathrm{E}\left|\varepsilon_{k}\right|^{2}$, we can obtain a set of linear equations by using the orthogonality principle in the mean-square estimation [17]. That is, the coefficients $\left\{r_{p}\right\}$ are selected to make the error $\varepsilon_{k}$ orthogonal to the signal samples $\left\{z_{k-p}\right\}$, i.e., $\mathrm{E}\left[\varepsilon_{k} z_{k-p}\right]=0$ for $0 \leq p \leq S-1$. Then, by using (11) and (26), the following set of equations are found

$$
\mathrm{E}\left[\left(a_{k-d}-\sum_{q=0}^{S-1} r_{q} z_{k-q}\right) z_{k-p}\right]=0, \quad p=0,1, \ldots, S-1
$$

or, equivalently,

$$
\sum_{q=0}^{S-1} r_{q} \mathrm{E}\left[z_{k-q} z_{k-p}\right]=\mathrm{E}\left[a_{k-d} z_{k-p}\right], \quad p=0,1, \ldots, S-1
$$

We use $\Gamma_{p q}=\mathrm{E}\left[z_{k-q} z_{k-p}\right]$ and $\xi_{p}=\mathrm{E}\left[a_{k-d} z_{k-p}\right]$ for a compact notation. After taking the expectations, we get

$$
\begin{aligned}
\Gamma_{p q} & =\sum_{m=0}^{I} y_{m} y_{m+p-q}+\sigma_{k-p}^{2} \delta_{p q}, & p, q=0, \ldots, S-1 \\
\xi_{p} & =y_{d-p}, & p=0,1, \ldots, S-1
\end{aligned}
$$




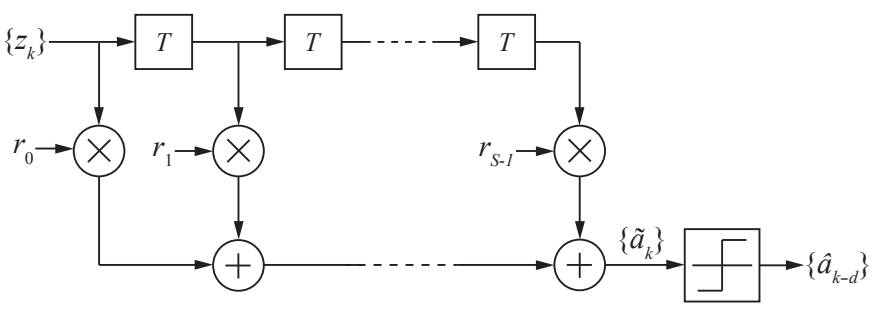

Fig. 5. The block diagram of MMSE equalizer with $S$ tap coefficients.

where $\delta_{p q}$ is the Kronecker delta function, $\sigma_{k-p}^{2}$ is the variance of the concentration sample $z_{k-p}$, and $y_{d-p}$ is the $(d-p)$ th coefficient of the equivalent discrete time channel. Thus, (28) can be written as

$$
\sum_{q=0}^{S-1} \Gamma_{p q} r_{q}=\xi_{p}, \quad p=0,1, \ldots, S-1
$$

Unlike the additive stationary Gaussian noise, the variance of the noise is signal-dependent in the diffusion-based MC. As a result, for different transmitted information sequences, there is a different optimum solution for the MMSE equalizer tap weights. The detected bits $\hat{a}_{k}$ through $\hat{a}_{k-S-I+1}$ are required to evaluate the variances $\sigma_{k}^{2}$ through $\sigma_{k-S+1}^{2}$ in (29), assuming the decisions are correct. Hence, the output of the decision device is used in the MMSE equalizer to solve (30) which makes the proposed MMSE equalizer signal sensitive. That is, the equalizer tap coefficients are adaptively updated based on the detected bits. Since using $\hat{a}_{k}$ in the estimation of $a_{k}$ is inconsistent, we set $\hat{a}_{k}=\frac{1}{2}$ in the estimation of $a_{k}$ as an ad hoc method to prevent this inconsistency. $\hat{a}_{k}=\frac{1}{2}$ has equal Euclidian distances to both $\hat{a}_{k}=0$ and $\hat{a}_{k}=1$, that is, $a_{k}$ can be 0 or 1 with equal probabilities, which is consistent with the equally likely transmitted bits. Also note that, the use of the wrong decisions causes error propagation which may deteriorate the performance of the MMSE equalizer

It is convenient to use matrix form to solve the set of equations given in (30) as follows

$$
\boldsymbol{\Gamma} \underline{\boldsymbol{r}}_{\mathrm{opt}}=\underline{\boldsymbol{\xi}}
$$

where $\underline{\boldsymbol{r}}_{\mathrm{opt}}$ is the column vector of $S$ optimum equalizer tap weights $\left\{r_{p}\right\}$ given as $\underline{\boldsymbol{r}}_{\mathrm{opt}}=\left[r_{0}, r_{1}, \ldots, r_{S-1}\right]^{\mathrm{T}}$ and $\boldsymbol{\Gamma}$ denotes the Hermitian covariance matrix with elements $\Gamma_{p q}$ given in (29), and $\boldsymbol{\xi}$ is the column vector with elements $\xi_{p}$ given in (29). Therefore, for $k$ th received signal $z_{k}$, the optimum equalizer tap weights to estimate $\tilde{a}_{k}$ are found as

$$
\underline{\boldsymbol{r}}_{\mathrm{opt}}=\boldsymbol{\Gamma}^{-1} \underline{\boldsymbol{\xi}}
$$

where $\boldsymbol{\Gamma}^{-1}$ is the inverse of the matrix $\boldsymbol{\Gamma}$. Due to the nonstationary characteristic of the noise, the optimum tap coefficients of the MMSE equalizer are updated at each sample using the previously detected bits. Thus, the MMSE equalizer needs to perform the operation given in (32) for each sample, which increases the complexity of the equallizer. However, the proposed method for the MMSE equalizer is not as complex as the sequence detection methods described in Section III.

\section{Decision-FeEdBACK Equalizer}

In this section, we consider the decision-feedback equalizer (DFE) which is a nonlinear type of equalizer to mitigate the ISI. The DFE is also suboptimum; however, its performance is generally better than that of suboptimum linear equalizers, e.g., the MMSE equalizer [17]. Furthermore, the DFE still is not as complex as the optimum ML and MAP sequence detection methods. The DFE consists of two sections that are a feedforward filter and a feedback filter as shown in Fig. 6 . The decisions are made on the output of the equalizer and they are propagated through the feedback filter to eliminate the ISI. Since the input of the feedback filter is the output of a decision device, the DFE has a nonlinear characteristics. The output of the DFE is the estimate of the transmitted bit and it is given by

$$
\tilde{a}_{k}=\sum_{q=0}^{L-1} f_{q} z_{k-q}+\sum_{q=1}^{M} b_{q} \hat{a}_{k-q-1}
$$

where $z_{k}$ is the noisy and ISI-distorted signal at the input of the DFE and $\hat{a}_{k}$ is the $k$ th detected bit.

The feedforward filter is based on the MMSE criterion and as analyzed in Section IV, its coefficients $f_{0}$ through $f_{L-1}$ are given by the system of linear equations

$$
\sum_{q=0}^{L-1} \Psi_{p q} f_{q}=\varphi_{p}, \quad p=0,1, \ldots, L-1
$$

where $\Gamma_{p q}$ and $\varphi_{p}$ are defined as

$$
\begin{aligned}
\Psi_{p q} & =\sum_{m=0}^{I} y_{m} y_{m+p-q}+\sigma_{k}^{2} \delta_{p q}, & p, q=0, \ldots, L-1 \\
\varphi_{p} & =y_{1-p}, & p=0,1, \ldots, L-1
\end{aligned}
$$

As stated in Section IV, the coefficients of the feedforward filter are found by using the matrix form representation of the system of the linear equations given in (34) as follows

$$
\underline{f}_{\mathrm{opt}}=\Psi^{-1} \underline{\varphi}
$$

where $\underline{f}_{\text {opt }}$ is the vector containing the optimum equalizer tap weights $\left\{f_{q}\right\}$, i.e., $\boldsymbol{f}_{\text {opt }}=\left[f_{0}, f_{1}, \ldots, f_{L-1}\right]^{\mathrm{T}}, \boldsymbol{\Psi}^{-1}$ is the inverse of the Hermitian covariance matrix with elements $\Psi_{p q}$ given in (35), and $\varphi$ is the column vector with elements $\varphi_{p}$ given in (35). As in the case of the MMSE equalizer, the optimum coefficients for the feedforward filter of the DFE are evaluated and updated at each sampling time.

The coefficients of the feedback filter of the DFE detector are expressed in terms of the coefficients of the feedforward filter [17] as follows

$$
b_{k}=-\sum_{q=0}^{L-1} f_{q} y_{k-q}=-\underline{\boldsymbol{f}}_{\mathrm{opt}}^{\mathrm{T}} \underline{\boldsymbol{y}}_{k}, \quad k=1,2, \ldots, M
$$

where $\underline{\boldsymbol{y}}_{k}$ is the $L$-dimensional column vector defined as $\underline{\boldsymbol{y}}_{k}=$ $\left[y_{k}, y_{k-1}, \ldots, y_{k-L+1}\right]^{\mathrm{T}}$. The values of the coefficients of the feedback filter $b_{1}$ through $b_{M}$ completely eliminate the ISI caused by the previously transmitted bits provided that the previous decisions are correct and that $M=L+I-1$ [22]. 


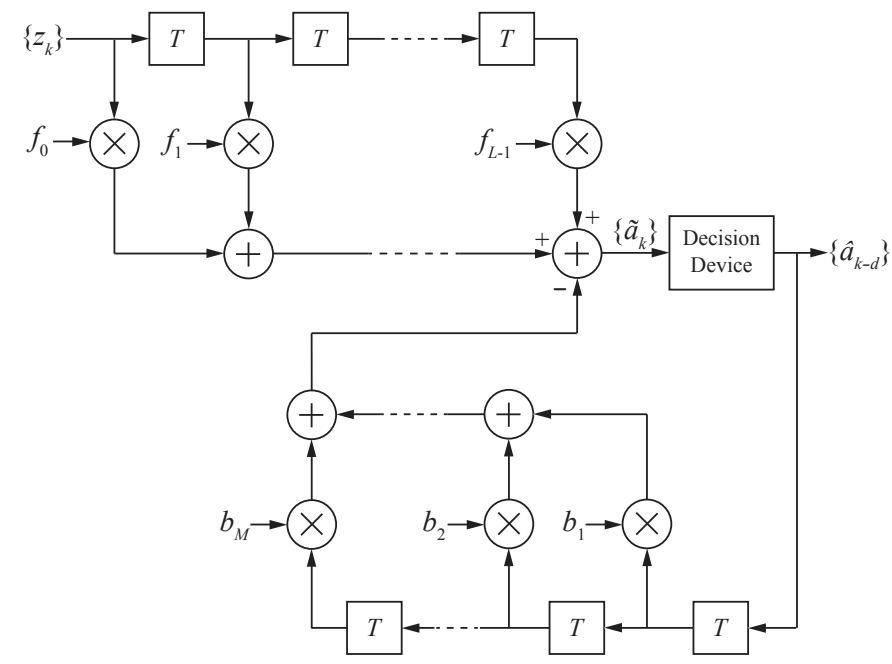

Fig. 6. The block diagram of decision-feedback equalizer

We choose $L=I+1$ and $M=2 I$. Thus, for the coefficients of the feedback filter given in (37), the output of the DFE given in (33) reduces to

$$
\begin{aligned}
\tilde{a}_{k} & =f_{0} y_{1} a_{k-1}+\sum_{q=0}^{I} f_{q} n_{k-q} \\
& =\beta a_{k-1}+w_{k}
\end{aligned}
$$

where $w_{k}$ is the correlated Gaussian noise term and $\beta=f_{0} y_{1}$. The correlation length of the noise is the same as the ISI length $I+1$ which can be seen from (38).

In Section IV, the output of the MMSE equalizer is quantized by a decision device to the nearest bit in Euclidian distance. However, for a decision device of the DFE, we propose an alternative method to minimize the probability of the decision error. Motivated by the ML sequence detection, the decisions are made as follows

$$
\begin{aligned}
\hat{a}_{k-1} & =\arg \max _{a_{k-1}} f\left(\tilde{a}_{k} \mid \underline{\boldsymbol{a}}_{k-1}^{k}, \underline{\hat{\boldsymbol{a}}}_{k-I}^{k-2}, \underline{\tilde{\boldsymbol{a}}}_{k-I}^{k-1}\right) \\
& =-\arg \min _{a_{k-1}} \ln f\left(\tilde{a}_{k} \mid \underline{\boldsymbol{a}}_{k-1}^{k}, \underline{\hat{\boldsymbol{a}}}_{k-I}^{k-2}, \underline{\tilde{\boldsymbol{a}}}_{k-I}^{k-1}\right) \\
& =\arg \min _{a_{k-1}} \mathcal{H}\left(a_{k}, a_{k-1}\right)
\end{aligned}
$$

where $\mathcal{H}\left(a_{k}, a_{k-1}\right)$ is the comparison metric given by

$$
\mathcal{H}\left(a_{k}, a_{k-1}\right)=-\ln f\left(\tilde{a}_{k} \mid \underline{\boldsymbol{a}}_{k-1}^{k}, \underline{\hat{\boldsymbol{a}}}_{k-I}^{k-2}, \underline{\tilde{\boldsymbol{a}}}_{k-I}^{k-1}\right)
$$

where $\mathcal{H}(0,0), \mathcal{H}(0,1), \mathcal{H}(1,0)$, and $\mathcal{H}(1,1)$ are the metric values of the possible combinations of $a_{k}$ and $a_{k-1}$. In this case, the input samples of the decision device, i.e. $\tilde{a}_{k}$ 's, are not independent due to the correlated noise term $v_{k}$. By Bayes rule, the pdf given in (39) can be written as

$$
f\left(\tilde{a}_{k} \mid \underline{\boldsymbol{a}}_{k-1}^{k}, \underline{\hat{\boldsymbol{a}}}_{k-I}^{k-2}, \underline{\tilde{\boldsymbol{a}}}_{k-I}^{k-1}\right)=\frac{f\left(\underline{\tilde{\boldsymbol{a}}}_{k-I}^{k} \mid \underline{\boldsymbol{a}}_{k-1}^{k}, \underline{\hat{\boldsymbol{a}}}_{k-I}^{k-2}\right)}{f\left(\underline{\tilde{\boldsymbol{a}}}_{k-I}^{k-1} \mid \underline{\boldsymbol{a}}_{k-1}^{k}, \underline{\hat{\boldsymbol{a}}}_{k-I}^{k-2}\right)}
$$

By using the pdf of the independent molecule concentration samples at the output of the channel, the expression given in
(41) can be written as

$$
\begin{aligned}
& \frac{f\left(\underline{\tilde{\boldsymbol{a}}}_{k-I}^{k} \mid \underline{\boldsymbol{a}}_{k-1}^{k}, \underline{\hat{\boldsymbol{a}}}_{k-I}^{k-2}\right)}{f\left(\underline{\tilde{\boldsymbol{a}}}_{k-1}^{k-1} \mid \underline{\boldsymbol{a}}_{k-1}^{k}, \underline{\boldsymbol{a}}_{k-I}^{k-2}\right)} \\
& =\sqrt{\frac{(2 \pi)^{I} \operatorname{det} \mathbf{c}_{k}}{(2 \pi)^{I+1} \operatorname{det} \mathbf{C}_{k}}} \frac{\exp \left[-\frac{1}{2} \mathbf{N}_{k}{ }^{\mathrm{T}} \mathbf{C}_{k}^{-1} \mathbf{N}_{k}\right]}{\exp \left[-\frac{1}{2} \mathbf{n}_{k}{ }^{\mathrm{T}} \mathbf{c}_{k}^{-1} \mathbf{n}_{k}\right]}
\end{aligned}
$$

where $\mathbf{C}_{k}$ is the $(I+1) \times(I+1)$ covariance matrix of the data samples $\underline{\tilde{\boldsymbol{a}}}_{k-I}^{k}$ given $\underline{\boldsymbol{a}}_{k-1}^{k}, \underline{\hat{\boldsymbol{a}}}_{k-I}^{k-1}$ and the $\mathbf{c}_{k}$ is the $I \times I$ lower principal submatrix of

$$
\mathbf{C}_{k}=\left[\begin{array}{cc}
\cdot & \cdot \\
\cdot & \mathbf{c}_{k}
\end{array}\right]
$$

$\mathbf{N}_{k}$ is the $(I+1)$-dimensional column vector of the differences between $\tilde{a}_{k}$ 's and their mean values given $\underline{\boldsymbol{a}}_{k-1}^{k}, \underline{\hat{\boldsymbol{a}}}_{k-I}^{k-2}$, i.e.,

$$
\mathbf{N}_{k}=\left[\left(\tilde{a}_{k}-\beta a_{k-1}\right), \ldots,\left(\tilde{a}_{k-I-1}-\beta \hat{a}_{k-I}\right)\right]^{\mathrm{T}}
$$

$n_{k}$ is the vector including the last $I$ elements of $\mathbf{N}_{k}$, i.e.,

$$
\mathbf{n}_{k}=\left[\left(\tilde{a}_{k-1}-\beta \hat{a}_{k-2}\right), \ldots,\left(\tilde{a}_{k-I}-\beta \hat{a}_{k-I-1}\right)\right]^{\mathrm{T}}
$$

Using (42) for the comparison metric calculation and cancelling constant terms, we get

$$
\mathcal{H}\left(a_{k}, a_{k-1}\right)=\ln \frac{\operatorname{det} \mathbf{C}_{k}}{\operatorname{det} \mathbf{c}_{k}}+\mathbf{N}_{k}^{\mathrm{T}} \mathbf{C}_{k}^{-1} \mathbf{N}_{k}-\mathbf{n}_{k}^{\mathrm{T}} \mathbf{c}_{k}^{-1} \mathbf{n}_{k}
$$

Therefore, the decision strategy of the decision device is

$\hat{a}_{k-1}= \begin{cases}1, & \text { if } \min \mathcal{H}\left(a_{k}, a_{k-1}\right)=\{\mathcal{H}(0,1) \text { or } \mathcal{H}(1,1)\} \\ 0, & \text { if } \min \mathcal{H}\left(a_{k}, a_{k-1}\right)=\{\mathcal{H}(0,0) \text { or } \mathcal{H}(1,0)\}\end{cases}$

The decision device computes the comparison metric given in (46) for each decision; thus, such a decision device is more complex than a simple quantizer as used for the MMSE equalizer in Section IV. In the performance analysis, we analyze the improvement in the performance of the DFE after implementing a decision device that uses the metric given (46).

\section{AdAPTIVE RECEIVER}

To recover the transmitted bits from the molecule concentration samples distorted by both the ISI and noise, we present the following methods: the MAP and ML sequence detectors, MMSE equalizer and DFE in the previous sections. For each case, the RN needs the knowledge of the discretetime equivalent channel coefficients of the diffusion-based MC channel, i.e., $y_{0}$ through $y_{I}$. However, the molecular diffusion channel is time-varying caused by the time variation of the diffusion coefficient $D$. Therefore, even if the RN knows the initial channel coefficients, since the molecular diffusion channel is time-varying, initial knowledge of the $\mathrm{RN}$ is not useful after the channel coefficients change.

To find the discrete-time equivalent coefficients of a channel that is unknown or time varying, we adopt a channel estimator presented in [17], [18]. The channel estimator of the RN recursively estimates the tap coefficients of the channel to minimize the MSE between the actual received sequence and the output of the estimator. That is, the channel estimator approximates the actual discrete-time equivalent channel with a similar structure to the actual channel. The steepest-descent 


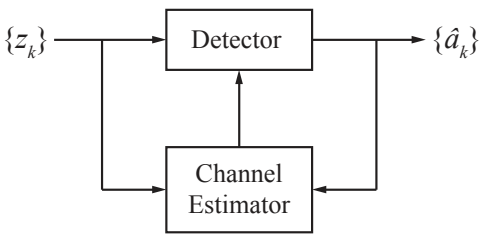

Fig. 7. The conceptual model of the channel estimator using in a detector.

algorithm can be used for the estimation of the channel tap coefficients. The operation of the channel estimator is as follows. Firstly, at each sampling time a new data sample $z_{k}$ and the detected information bit for the corresponding data sample $\hat{a}_{k}$ are fed to the channel estimator as illustrated in Fig. 7. The estimate for the data sample $\tilde{z}_{k}$ is obtained by multiplying the sequence of detected bits $\left\{\hat{a}_{j}\right\}$ by the corresponding estimate of the coefficient $\left\{\tilde{y}_{j}\right\}$ and summing the results of all multiplications,

$$
\tilde{z}_{k}=\sum_{j=0}^{I} \tilde{y}_{j} \hat{a}_{k-j}, \quad k=0,1, \ldots
$$

The difference between the data sample $z_{k}$ and its estimate $\tilde{z}_{k}$ at $k$ th iteration step generates an error signal $\varepsilon^{(k)}=z_{k}-\tilde{z}_{k}$. To form a new set of estimates of the channel coefficients, the obtained error is used as

$$
\tilde{y}_{j}^{(k+1)}=\tilde{y}_{j}^{(k)}+\Delta \varepsilon^{(k)} \hat{a}_{k-j}, \quad j=0,1, \ldots, I-1
$$

where $\tilde{y}_{j}^{(k)}$ is the estimate of the channel coefficient $y_{j}$ at the $k$ th iteration step, $\Delta$ is the step size, and $\hat{a}_{j}$ is the $j$ th detected information bit in the estimator. The speed of the convergence and the accuracy of the estimation are controlled by the value of the step size $\Delta$. A delay equal to the decision delay of the transmitted bit is required to have a proper synchronization. When the MSE between $z_{k}$ and $\hat{z}_{k}$ is minimized, the estimates of the tap weight coefficients of the channel estimator are exactly equal to the equivalent discrete-time channel coefficients [17]. Furthermore, since the steepest descent algorithm is used in (49) to adjust the channel coefficient estimates, the channel estimator is able to track slow variations in the actual channel coefficients. We assume that the time variation of the molecular diffusion channel is slow compared to the convergence speed of the channel estimator. The channel estimator introduced in this section is compatible with all detectors presented above.

\section{Performance Analysis}

In this section, we present the performance analysis of the ML sequence detector, the MMSE equalizer and the DFE detector. Since we assume that the transmitted information bits are equally likely, the MAP sequence detection is equivalent to the ML sequence detection. For the performance comparison of the proposed methods, the bit error rates of the corresponding methods are considered. We use the Monte Carlo simulation to estimate the bit error rates. The bit error rates of the proposed detectors are evaluated with respect to the signal to noise ratio (SNR) at the RN which is defined as

$$
\mathrm{SNR}=\frac{P_{y}}{P_{n}}=\frac{\frac{1}{I+1} \sum_{n=0}^{I}\left|y_{n}\right|^{2}}{\mathrm{E}\left[\sigma_{k}^{2}\right]}=\frac{\frac{1}{I+1} \sum_{n=0}^{I}\left|y_{n}\right|^{2}}{\frac{0.5}{V_{R}} \sum_{n=0}^{I} y_{n}}
$$

where $P_{y}$ and $P_{n}$ are considered as the received signal power and the noise power, respectively, at the receiver. In the SNR definition, we use the expected value of the noise variance. As seen from (50), SNR depends on only the discrete time equivalent channel coefficients. Therefore, we change the amplitude of the concentration pulse $A$ to change SNR values.

For an emitted concentration pulse, the noiseless concentration in the reception space $y(t)$ reaches its maximum at $t=t_{\max }$ as stated above. Hence, we set the sampling time of $\mathrm{RN}$ as $T=t_{\max }$ in order to sample the concentration with the highest SNR for a given concentration pulse amplitude $A$. Furthermore, the change in $y(t)$ at different communication distances causes $t_{\max }$ to change. Thus, the signaling interval $T=t_{\max }$ and the information transmission rate $R=1 / T$ change with the communication distance. In the analysis, we set the molecule emission duration as

$$
T_{e}=\frac{r^{2}}{6 D_{0}}
$$

where $D_{0}$ is the initial value of the diffusion coefficient $D$. The value of the $T_{e}$ effects the ISI length and the signaling rate. The selection of the molecule emission duration depends on the application of the MC and the duration can be set in different ways. Since, we use the expression given in (51), the ISI length can be considered as $I+1=6$ because $y(k T)$ for $k>6$ becomes sufficiently small that can be ignored. Moreover, in the performance analysis, we use $2^{5}$-state trellis for the ML detector, $S=6$ taps for the MMSE equalizer, $L=6$ taps for the feedforward filter of the DFE and $M=10$ tap for the feedback filter of the DFE. In the analysis, we set the radius the spherical reception space $\rho=1 \mathrm{~nm}$.

\section{A. Time Invariant Channel}

In this section, we consider the diffusion-based MC channel as time invariant by setting $D=2.2 \times 10^{-9} \mathrm{~m}^{2} / \mathrm{s}$ and we assume that the channel is known at RN. The performances of the ML sequence detector, the MMSE equalizer, the DFE using the decision metric given in (46), and the DFE using the simple quantizer are analyzed. In Fig. 8(a), (b) and (c), bit error rates of the proposed detectors with respect to SNR for the communication distances $r=50 \mathrm{~nm}, r=250 \mathrm{~nm}$, and $r=1 \mu m$, respectively, are illustrated. We do not show the ML sequence detector performance beyond $\mathrm{SNR}=13 \mathrm{~dB}$ since the Monte Carlo simulation takes too long time to compute. In the simulations, the signaling rates $R=3.12 \mathrm{Mbps}$ for $r=$ $50 \mathrm{~nm}, R=127.9 \mathrm{kbps}$ for $r=250 \mathrm{~nm}$, and $R=8.1 \mathrm{kbps}$ for $r=1000 \mathrm{~nm}$ are used. The first conclusion can be drawn from Fig. 8 is that for different distances, the performances of the considered methods are very similar. This is an expected result since the communication rates are adjusted at different 

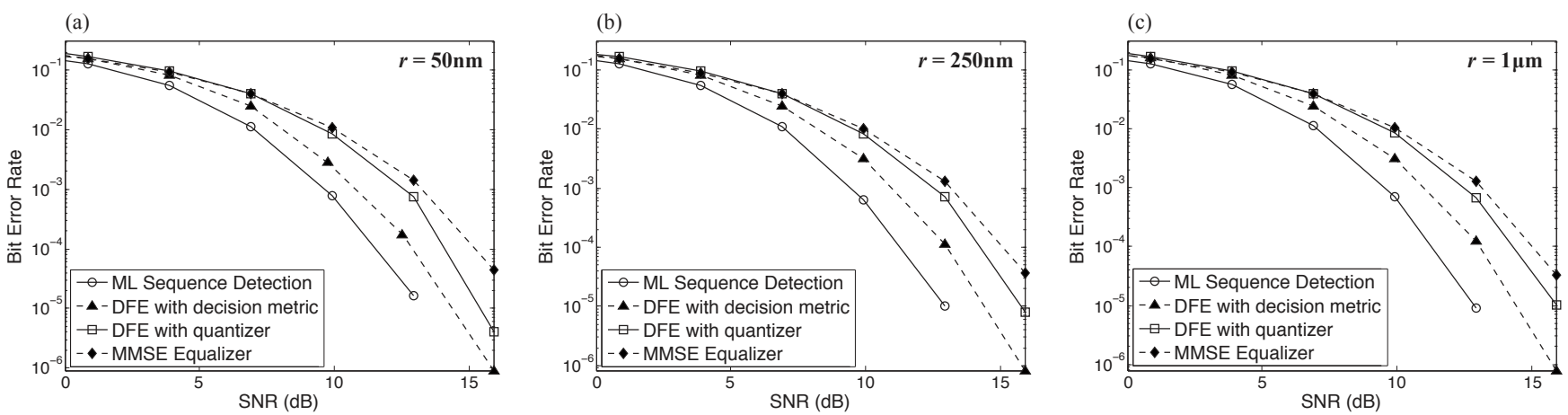

Fig. 8. Bit error rates of four different detection approaches at different communication distances (a) $r=50 \mathrm{~nm}, R=3.12 \mathrm{Mbps}$, (b) $r=250 \mathrm{~nm}, R=$ $127.9 \mathrm{kbps}$, and (c) $r=1 \mu \mathrm{m}, R=8.1 \mathrm{kbps}$ with same time invariant diffusion-based molecular channel known at RN.

(a)

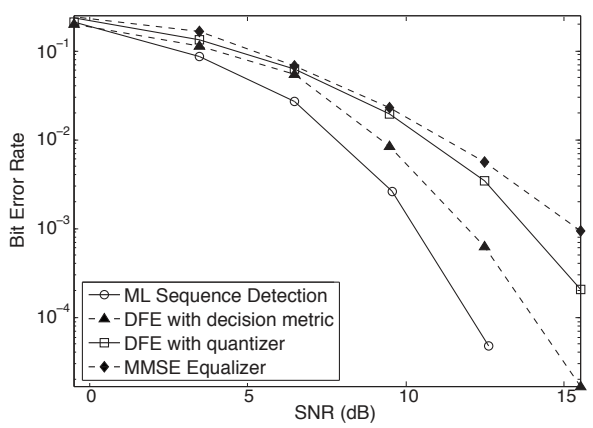

(b)

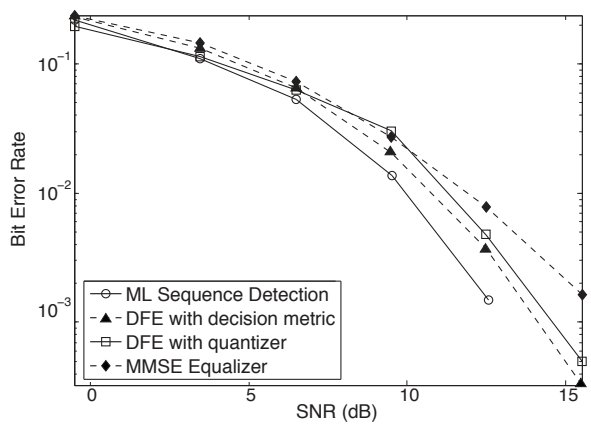

Fig. 9. Bit error rates of four different detection methods for time varying diffusion-based molecular communication channels with $\left(\right.$ a) $D(t)=2.2 \times 10^{-9}+$ $0.8 \times 10^{-9} \cos (2 \pi t) \mathrm{m}^{2} / \mathrm{s}$ and (b) $D(t)=2.2 \times 10^{-9}+0.8 \times 10^{-9} \cos (10 \pi t) \mathrm{m}^{2} / \mathrm{s}$.

communication distances in order to sample the concentration with the highest SNR for a given concentration amplitude $A$. Therefore, the same bit error rate can be achieved at a longer distance with a lower transmission rate.

Since the ML sequence detector is the optimum receiver, in the sense that it minimizes bit error probability of a sequence [17], it gives the best performance compared to the rest of the methods as seen in Fig. 8. On the other hand, the MMSE equalizer has the worst performance among the others. The DFE detectors have better performance than the MMSE equalizer as expected. Furthermore, the DFE with the decision device at the output is almost $2 \mathrm{~dB}$ better than the DFE with a simple quantizer at the output for a bit error rate equal to $10^{-4}$. For SNR less than $10 \mathrm{~dB}$, the performances of the DFE and MMSE equalizer are very close to each other. Then, we can conclude that using a feedback filter does not improve much the performance of the forward MMSE equalizer at low SNR values in the MC. Moreover, the performances of the proposed methods increase with an increase in the complexity. That is, the best performance is obtained by the ML detector which is the most complex detector and the MMSE equalizer which is the most simple detector gives the worst bit error rate.

\section{B. Time Varying Channel}

The diffusion-based MC channel has time varying characteristics depending on several factors as stated in Section II. In this part, we use the time varying diffusion channel together with the channel estimator described in Section VI with a step size $\Delta=0.001$ to investigate the performances of the proposed methods in more realistic conditions. In this part, we set the signaling rate $R=8.1 \mathrm{kbps}$ at a communication distance $r=250 \mathrm{~nm}$ for the simulations. In the Fig. 9(a) and (b), the performance analyses of the proposed methods for $D(t)=2.2 \times 10^{-9}+0.8 \times 10^{-9} \cos (2 \pi t) \mathrm{m}^{2} / \mathrm{s}$ and $D(t)=2.2 \times 10^{-9}+0.8 \times 10^{-9} \cos (10 \pi t) \mathrm{m}^{2} / \mathrm{s}$, respectively, are shown. The time variation of the first channel is 5 times slower than the time variation of the second channel. The performances of all methods decrease when the time varying channel is used, which can be seen when we compare Fig. 8(b) and Fig. 9. Although we use the channel estimator for the time varying channel, the detectors cannot exactly know the channel. That is, the transmitted information is recovered based on an estimate of the time varying channel coefficients which might decrease the performance of the proposed methods. Moreover, the convergence speed of the channel estimator should be fast enough to estimate the exact channel coefficients. The step size $\Delta$ can be adjusted according to the time variation characteristic of the channel. The performances of the proposed methods for a time varying channel whose time variation is faster can be seen in Fig. 9(b). We can say that the performances of all methods decrease with an increase in the time variation of the channel if the step size $\Delta$ is not adjusted accordingly.

\section{CONCLUSION}

In the MC, high signaling rates causes ISI because of the infinite duration impulse response of the diffusion-based 
molecular channel. In addition, the MC suffers from a signaldependent noise at the receiver. For the first time in the literature, four signal detection methods for nanodevices communicating via diffusion-based molecular communication channel are proposed in this paper. We present the sequence detection methods based on MAP and ML criterions, the MMSE equalizer, and the DFE to mitigate both the ISI and noise in the MC. Firstly, the diffusion-based molecular channel is described, the impulse response of the channel is obtained. Then, the nonstationary particle counting noise is incorporated to the model to design the detection methods properly. Succeeding that we present the MAP and ML sequence detection methods and derive branch metrics of the trellis search in the Viterbi algorithm. Next, a linear equalizer based on MMSE criterion is introduced. Then, a nonlinear type equalizer, DFE, is presented. To improve the performance of the DFE, we propose a decision device based on ML detection method at the output of the DFE.

According to the simulation results, while the best performance is achieved by the ML sequence detector which is the most complex detector, the MMSE equalizer has the worst performance which is the most simple detector. The performance of the DFE stands between MMSE equalizer and ML sequence detector. Furthermore, the usage of a decision device instead of a simple quantizer increases the performance of the DFE. A detector can be selected based on the required reliability of the communication and the feasible complexity of the detector at nanoscale. Moreover, since the diffusion-based molecular channel is time varying, we propose using a channel estimator at $\mathrm{RN}$. Our results reveal that in $\mathrm{MC}$, very high information transmission rates can be achieved with very low bit error rates by using the detection methods presented above. Thus, the detection methods obtained in this paper significantly improves the communication capabilities of nanodevices.

\section{REFERENCES}

[1] S. Hiyama, Y. Moritani, T. Suda, R. Egashira, A. Enomoto, M. Moore, T. Nakano, "Molecular Communication," in Proc. of NSTI Nanotech. 2005, Anaheim, California, USA, 2005.

[2] I. F. Akyildiz, F. Brunetti, C. Blazquez, "Nanonetworks: A new communication paradigm," Comp. Net., vol. 52, no. 12, pp. 2260-2279, 2008.

[3] L. Parcerisa Giné, I. F. Akyildiz, "Molecular communication options for long range nanonetworks," Computer Networks (Elsevier), vol. 53, no. 16, pp. 2753-2766, Nov 2009.

[4] M. Gregori, I. F. Akyildiz, "A new nanonetwork architecture using flagellated bacteria and catalytic nanomotors," IEEE J. Sel. Areas Commun., vol. 28, no. 4, pp. 612-619, 2010.

[5] B. Atakan, S. Balasubramaniam, O. B. Akan, "Body Area NanoNetworks with Molecular Communications in Nanomedicine," IEEE Communications Magazine, vol. 50, no. 1, pp. 28-34, Jan 2012.

[6] M. Pierobon, I. F. Akyildiz, "Diffusion-based noise analysis for molecular communication in nanonetworks," IEEE Transactions on Signal Processing, vol. 59, 2532-2547, 2011.

[7] L. P. Giné, I. F. Akyildiz, "Molecular communication options for long range nanonetworks," Computer Networks, vol. 53, pp. 2753-66, 2009.

[8] D. Kilinc, O. B. Akan, "An Information Theoretical Analysis of Nanoscale Molecular Gap Junction Communication Channel Between Cardiomyocytes," to appear in IEEE Trans. Nanotechnol., 2013.

[9] B. J. MacLennan, "Morphogenesis as a model for nano communication," Nano Communication Networks, vol. 1, no. 3, pp. 199-208, 2010.

[10] M. Pierobon, I. F. Akyildiz, "Information Capacity of Diffusion-based Molecular Communication in Nanonetworks," in Proc. of IEEE Int. Conf. on Comp. Comm, INFOCOM, Miniconf., China, April 2011.
[11] B. Atakan, O. B. Akan, "Deterministic Capacity of Information Flow in Molecular Nanonetworks," Nano Communication Networks Journal (Elsevier), vol. 1, no. 1, pp. 31-42, March 2010.

[12] T. Nakano, J. Q. Liu, "Design and analysis of molecular relay channels: An information theoretic approach," IEEE Transactions on Nanobioscience, vol. 9, no. 3, pp. 213-221, Jun 2009.

[13] A. Einolghozati, M. Sardari, F. Fekri, "Capacity of diffusion based MC with ligand receptors, in 2011 IEEE Information Theory Workshop.

[14] M. Pierobon, I. F. Akyildiz, "A physical end-to-end model for molecular communication in nanonetworks,' IEEE JSAC, vol. 28, pp. 602-611, 2010.

[15] B. Atakan, O. B. Akan, "On molecular multiple-access, broadcast, and relay channels in nanonetworks," in Proc. 3rd Int. Conf. Bio-Inspired Models Netw., Inf., Comput. Syst., 2008, Art. no. 16.

[16] B. Atakan, O. B. Akan, "An information theoretical approach for molecular communication," in Proc. 2nd Int. Conf. Bio-Inspired Models Netw., Inf., Comput. Syst., 2007, pp. 33-40.

[17] J. G. Proakis, Digital Communications, 5th ed. New York: McGrawHill, 2001.

[18] F. Magee, J. Proakis, "Adaptive maximum-likelihood sequence estimation for digital signaling in the presence of intersymbol interference (Corresp.)," IEEE Trans. Inf. Theory, vol.19, pp. 120-124, 1973.

[19] A. Kavcic, J. M. F. Moura, "The Viterbi algorithm and Markov noise memory," IEEE Trans. Inf. Theory, vol.46, pp.291-301, 2000.

[20] A. Kavcic, J. M. F. Moura, "Correlation-sensitive adaptive sequence detection," IEEE Trans. Magn., vol. 34, no.3, pp.763-771, 1998.

[21] B. Sklar, "How I learned to love the trellis," IEEE Signal Processing Magazine, vol.20, no.3, pp. 87-102, May 2003.

[22] G. L. Stuber, Principles of Mobile Communication, 3rd ed. Kluwer Academic Publishers, Boston, 1996

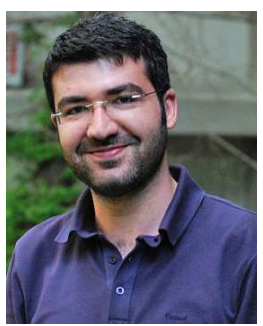

Deniz Kilinc received his B.Sc. degree in Electrical and Electronics Engineering from Middle East Technical University, Ankara, Turkey, in 2011. He is currently a research assistant in the Next-generation and Wireless Communication Laboratory and pursuing his M.Sc. degree at the Electrical and Electronics Engineering Department, Koc University, Istanbul, Turkey. His current research interests include nanoscale communication, intra-body communication, and detection \& estimation theory.

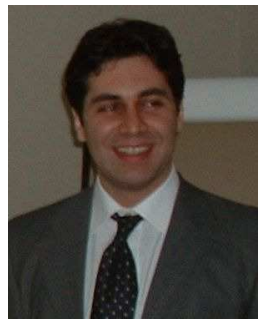

Ozgur B. Akan [M'00, SM'07] (akan@ku.edu.tr) received his $\mathrm{Ph} . \mathrm{D}$. degree in electrical and computer engineering from the Broadband and Wireless Networking Laboratory, School of Electrical and Computer Engineering, Georgia Institute of Technology in 2004. He is currently a full professor with the Department of Electrical and Electronics Engineering, Koc University and the director of the Next-generation and Wireless Communications Laboratory. His current research interests are in wireless communications, nano-scale and MCs, and information theory. He is an Associate Editor of IEEE Transactions on Vehicular Technology, International Journal of Communication Systems (Wiley), and Nano Communication Networks Journal (Elsevier). He is currently General Co-Chair of ACM MOBICOM 2012, IEEE MoNaCom 2012, and TPC CoChair of IEEE ISCC 2012. 